Material and Methods: All simple myringoplasty of the last 3 years have been revaluated. Exclusion criteria were the presence of a cholesteatoma and chronic otitis. An otoscopic picture of both ears was taken for each patient. The site of perforation was classified into anterior, posterior and subtotal. A PTA, according to the guidelines of the AAOHNS has been performed before and 2 months after surgery.

Results: A total of 123 patients undergoing simple myringoplasty was identified. In 33 patients we used $\mathrm{C}$, in $33 \mathrm{~F}$ and in $26 \mathrm{P}$. The overall failure rate was $10 \%$, divided in: $12.1 \%$ for $\mathrm{C}$ (plus a further $12.1 \%$ of microperforation all repaired), $2.7 \%$ for F, $18.2 \%$ for P. The status of the contralateral ear showed it was pathological in $48.5 \%$ of cases of $\mathrm{C}$, $16.6 \% \mathrm{~F}$ and $18.2 \%$ of $\mathrm{P}$. The site of the perforation was anterior in $48.5 \%$ of $\mathrm{C}, 41.6 \%$ of $\mathrm{F}$ and $40.1 \%$ of $\mathrm{P}$; posterior in $12.1 \%$ of C, $13.8 \%$ of $\mathrm{F}$ and $45.4 \%$ of $\mathrm{P}$; subtotal in $39.4 \%$ of $\mathrm{C}, 44.4 \mathrm{~F}$ and $13.6 \%$ of $\mathrm{P}$. The ABG was $26.9 \mathrm{~dB}$ for the preoperative $\mathrm{C}, 20.7 \mathrm{~dB}$ for $\mathrm{F}$ and $18.6 \mathrm{~dB}$ for and $\mathrm{P}$; The postoperative $\mathrm{ABG}$ was $17.3 \mathrm{~dB}$ for $\mathrm{C}, 13.1 \mathrm{~dB}$ for $\mathrm{F}$ and $11.5 \mathrm{~dB}$ for $\mathrm{P}$. The auditive gain (difference of $\mathrm{ABG}$ pre and postop) was $9.5 \mathrm{~dB}$ for $\mathrm{C}, 7.5 \mathrm{~dB}$ for $\mathrm{F}$ and $7 \mathrm{~dB}$ for $\mathrm{P}$.

Conclusions: The results show an overall success rate in line with the literature. It emerges that $\mathrm{F}$ has the best success rate but $\mathrm{C}$ is used mostly in cases where the contralateral ear is pathological. The auditory gain is comparable, even if $\mathrm{C}$ is chosen in the cases with a worse initial ABG.

doi:10.1017/S0022215116006198

\section{ID: IP123}

\section{An evaluation of the NHS Clinical Commissioning Policy on Bone Anchored Hearing Aids}

Presenting Author: Rishi Mandavia

\author{
Rishi Mandavia ${ }^{1}$, Samit Unadkat ${ }^{2}$, Anne Schilder ${ }^{1}$ \\ ${ }^{1}$ Ear Institute, University College London, \\ ${ }^{2}$ London North Thames ENT Registrar
}

\section{Learning Objectives:}

Introduction: The NHS Commissioning Policy on Bone Anchored Hearing Aids (BAHA) identifies the criteria for the commissioning of BAHA services and therefore has a major impact on patient access to care. This paper aims to evaluate the evidence base informing the NHS Commissioning Policy on BAHAs. We also aim to produce recommendations on BAHA policy development.

Methods: This study was conducted in two parts.

1) Critical assessment of the evidence based informing the NHS Commissioning Policy on BAHAs. Quality of included articles and the overall strength of the policy were assessed using the Grading of Recommendations Assessment, Development, and Evaluation (GRADE) System.
2) Systematic review of the literature on BAHAs published since the release of NHS Commissioning Policy. Papers were included if they could be used to inform the Commissioning Policy on BAHAs.

Results: All studies referenced by the policy were graded as 'low quality' or 'very low quality' evidence. The strength of the overall policy was graded as weak. The literature cited by the Commissioning Policy contained several areas of disagreement with the Commissioning Policy itself.

Nineteen articles were included following systematic review. These studies identified six areas for development of the NHS Commissioning Policy for BAHAs: 1) BAHA implantation in children with unilateral hearing loss; 2) BAHA as an alternative to other surgical treatments; 3 ) The minimum number of BAHAs implanted by a centre each year; 4) Unilateral BAHA implantation in patients with less than profound sensorineural hearing loss; 5) Bilateral BAHA implantation in adults; 6) BAHA implantation in patients with osteogenesis imperfecta.

Conclusion: It is important that these areas are reviewed by the commissioning board to help ensure equitable access to BAHA services and that resources are allocated effectively. It is also clear that high quality research is urgently needed in this field to help inform national policy.

doi:10.1017/S0022215116006204

\section{ID: IP124}

\section{Couplers for Vibrant Soundbridge ${ }^{\circledR}$ \\ implant vs no-Coupler-Vibrant \\ Soundbridge ${ }^{\circledR}$ implant}

Presenting Author: Manuel Manrique

Manuel Manrique

$N A$

Introduction: The middle ear active implant Vibrant Soundbridge ${ }^{\circledR}$ (VSB) is a device designed for the treatment of the sensorineural, mixed and conductive hearing losses. Depending on the type of the hearing loss and the anatomical condition of the different middle ear structures, the placement of its FMT can be carried out in different ossicular chain points or directly on the round or oval window, aimed to obtain a direct stimulation of the inner ear. Recently, new Couplers have been designed to obtain a better coupling of the FMT with these structures.

Objectives: To compare surgical feasibility and auditory performance with VSB traditional system versus the new "Couplers" for the VSB implant

Methods and materials: Thirty eight patients treated with VSB systems are included at the moment. Eleven patients implanted with VSB Coupler versus 27 patients with no-Coupler VSB. Three out of eleven VSB Coupler implants were indicated for sensorineural hearing loss (SNHL) patients and eight of them for conductive and mixed hearing loss patients. Regarding no-Coupler VSB, seven patients were diagnosed of SNHL whereas twenty of conductive and mixed hearing loss patient. 
Mean thresholds for $0.5,1,2,3$ and $4 \mathrm{KHz}$ frequencies before and after implantation are obtained for $\mathrm{r}$ VSB Couple group and no-coupler VSB group. For SNHL air conduction is analysed whereas bone conduction is considered for conductive and mixed hearing loss.

Besides, functional gain and discrimination of disyllabic words at $65 \mathrm{~dB}$ in quiet is analyzed.

Results: Surgeon subjective perception is that Couplers simplify the surgical process, allow a more stable coupling and minimize the risks of post-surgical FMT movement as well. Focusing on SNHL patients, the mean hearing thresholds for the air conduction before and after the surgery were respectively $59.25 \mathrm{~dB}$ and $29.75 \mathrm{~dB}$ for the no-Coupler VSB group, opposite to $63 \mathrm{~dB}$ and $24.75 \mathrm{~dB}$ for the VSBCoupler group; and the mean functional gain was $30.25 \mathrm{~dB}$ for the no-Coupler VSB group, opposite to $38.25 \mathrm{~dB}$ for the VSB-Coupler group. In the disyllabic words test, difference was $78 \%$ for the no-Coupler VSB group and $82 \%$ for the VSB-Coupler group. For conductive-mixed hearing loss patients, mean hearing thresholds for the air conduction before and after the surgery were $84.75 \mathrm{~dB}$ and $85 \mathrm{~dB}$ respectively for the no-Coupler VSB group, opposite to $85 \mathrm{~dB}$ and $30.75 \mathrm{~dB}$ for the VSB-Coupler group; and mean functional gain was $47.5 \mathrm{~dB}$ for the no-Coupler VSB group, opposite to $53 \mathrm{~dB}$ for the VSB-Coupler group

Conclusions: Surgical procedure is simpler for coupler VSB for both indications (SNHL and conductive and mixed hearing loss). A greater tendency to improve auditory outcomes is described for VSB Coupler group vs no-Coupler VSB group.

doi:10.1017/S0022215116006216

\section{ID: IP125}

Aberrant internal carotid artery in the middle ear: a cause of aural fullness

\section{Presenting Author: Angels Martinez Arias}

Angels Martinez Arias ${ }^{1}$, Mario Prenafeta $^{2}$, Rosa Rosell ${ }^{2}$, Anton Aguila ${ }^{1}$, Mariana Campos ${ }^{1}$, Laura Samara ${ }^{1}$, Yolanda Escamilla ${ }^{1}$, Alda Cardesin ${ }^{1}$, Juan Jose Diaz ${ }^{1}$, Ricard Bargues ${ }^{1}$

${ }^{1}$ Hospital Parc Tauli Sabadell, Barcelona, Spain, ${ }^{2}$ Hospital Parc Tauli Sabadell,

Barcelona, Spain

Learning Objectives: Otologists should be aware of vascular malformations of the temporal bone. Aberrant ICA in the middle ear is a very rare finding and its damage during surgical procedures can lead to severe complications. When there is a suspicion of a middle ear vascular anomaly, CT scan of the temporal bone is the standard. It should be performed before any middle ear surgery, to avoid complications related to misdiagnosis. Endoscopic examination improves diagnosis of middle ear pathology.

Introduction: Aberrant internal carotid artery (ICA) in the middle ear is a rare vascular anomaly of the temporal bone and its diagnosis can be difficult because the symptoms and signs are often nonspecific.

Accidental injury during myringotomy or other middle ear surgeries, can lead to severe complications.
Methods: We report a case of a 47-year-old woman who complained of fullness in the right ear for 6 months, without hearing loss or tinnitus. She had no previous otological pathology.

Endoscopic otoscopy revealed a slight white-rosy mass behind the inferior half of the tympanic membrane.

A CT scan of the temporal bone confirmed aberrant ICA passing through the middle ear. A magnetic resonance angiography was also performed.

The patient was informed about the diagnosis and the possible complications of middle ear interventions, and regular follow-up was arranged.

Results: Color changes on otoscopic examination may suggest the presence of a vascular anomaly, as sometimes seen in aberrant ICA, due to its intratympanic course.

These features were seen in the CT scan: the ICA ran more laterally, there was an enhanced mass in the hypotympanum and a deficient bony plate along the tympanic portion of the ICA, bulging into the tympanic cavity.

The magnetic resonance angiography showed a lateralized right ICA with a reduced diameter.

Conclusions: Aberrant ICA in the middle ear should be identified before middle ear surgery, because misdiagnosis could lead to surgical complications: hemorrhage, stroke or death may occur if the vessel is damaged.

Clinical diagnosis is difficult because the symptoms and signs are nonspecific or absent and in some cases it can be discovered during middle ear surgery. However, symptoms such as pulsatile tinnitus or conductive hearing loss may be present.

Most authors recommend a conservative approach. If an asymptomatic aberrant ICA in the middle ear is diagnosed any physician involved in the patient's care should be informed.

doi:10.1017/S0022215116006228

\section{ID: IP126}

The impact of postoperative antrum pneumatization on hearing outcome after canal wall-down tympanoplasty with softwall reconstruction for cholesteatoma

\section{Presenting Author: Masatsugu Masuda}

Masatsugu Masuda, Jobe Matsumoto, Tatehiro Nakamura, Takehiro Matsuda, Kohno Naoyuki, Koichiro Saito

Kyorin University

Learning Objectives: It might be enough to concentrated on making the pneumatized cavity in the tympanic cavity alone. Further studies with a larger sample size will be needed to confirm it.

Introduction: It is not completely clear whether the pneumatized antrum is critical for good hearing in patients operated on for acquired cholesteatoma with canal wall-down type III (-c or -i) tympanoplasty with soft-wall reconstruction (CWDT-SWR). We thus investigated the correlation of pneumatization of the antrum with hearing outcome.

Methods: Eligible patients were: (1) CWDT-SWR was performed for the past four years; (2) ossiculoplasty was performed by the present author; (3) $>1$ year follow-up. Patients who had a past history of an otologic operation in 